\title{
Environmental effects on the performance of electrical grounding systems
}

\begin{abstract}
Effects of surrounding environment of grounding electrode locations on the performance of overall grounding system has been studied. Copper rods were buried at 25 different locations with various environmental settings and their respective variations in grounding resistance were monitored for up to 4 months. It was found that there is a clear dependence of grounding resistance on the condition of surrounding environment, in addition to the average soil resistivity. Installation of grounding electrodes near running waterways, huge trees as well as slopes should be avoided wherever possible as the ground resistance of electrodes in such environments may fluctuate significantly with time.
\end{abstract}

Keyword: Electrical grounding system; Surrounding environment; Grounding electrodes 